\title{
Estrategias de comercialización para el aumento de ventas en las urbanizaciones ubicadas en el cantón General Villamil Playas
} Marketing strategies for the increase of sales in the
urbanizations located in the canton General Villamil Playas

\author{
MSc, Burgos Benítez Giovanni ${ }^{1}$ \\ gburgos@ambiensa.com \\ MSc, Maridueña Arroyave Milton Rafael ${ }^{2}$ \\ rmaridueña@gmail.com
}

Recibido: 1/12/2018; Aceptado: 1/02/2019

\begin{abstract}
RESUMEN
El principal objetivo del presente artículo, es el de presentar estrategias de comercialización que tengan como resultado el aumento de ventas en las urbanizaciones ubicadas en el cantón General Villamil Playas de la provincia del Guayas. El aumento en el número de adultos mayores jubilados que residen en el cantón antes mencionado, fue una de las principales causas que motivaron al desarrollo de este artículo científico; además el mismo contribuirá a la mejora de la industria de la construcción, puesto que con la captación de más clientes, se verá fortalecida en la generación de ingresos, con la concepción de las acciones estratégicas de comercialización que se tomaron en cuenta. Se realizó una investigación exploratoria dirigida a los jubilados reconocidos por el Instituto Ecuatoriano de Seguridad Social (IESS), para identificar sus necesidades, preferencias y aceptación de vivir en una urbanización cerca del mar, los resultados del mismo indicaron que los adultos mayores si estarían dispuestos en vivir en una urbanización que presente beneficios para ellos, siendo así con la aplicación de estrategias de comercialización al correcto segmento de mercado, las ventas irían en aumento en las urbanizaciones privadas.
\end{abstract}

Palabras Clave: adultos mayores, comercialización, estrategias, marketing, urbanización

\footnotetext{
1 Universidad Tecnológica Empresarial de Guayaquil, Guayaquil, Ecuador.

2 Universidad Estatal Península de Santa Elena, Santa Elena, Ecuador.
} 


\begin{abstract}
The main objective of this article is to present marketing strategies that result in increased sales in the urbanizations located in the General Villamil Playas canton of the province of Guayas. The increase in the number of elderly people residing in the canton was one of the main causes that motivated the development of this scientific article and it will also contribute to the improvement of the construction industry, since with the acquisition of more clients, it will be strengthened in the generation of income, with the conception of strategic marketing actions that were taken into account. An exploratory research was conducted, aimed at people aged 65 years and older. The results indicated that older adults would be willing to live in an urbanization that presents benefits for them, thus being with the application of marketing strategies to the correct market segment, thereby sales would increase in private developments.
\end{abstract}

Keywords: older adults, trading, strategies, marketing, urbanization

\title{
Introducción
}

La construcción representa aproximadamente el 10\% del PIB de Ecuador. El principal inversor en el sector es el gobierno con una participación del $80 \%$ de la inversión total; el sector privado tiene una participación del 20\% (CCG, 2018) En los últimos años, el sector ha tenido un crecimiento excepcional en gran parte debido al aumento del gasto público en infraestructura. Para la década anterior a 2015, el sector de la construcción había crecido a una tasa anual promedio de $7.2 \%$ desde 2004, superando ampliamente el crecimiento promedio del PIB de $4.7 \%$ para ese período.

Desde 2007, los mercados inmobiliarios en los centros urbanos de Ecuador se apreciaron en torno al $10 \%$ antes de desacelerarse hacia fines de 2013. Para 2014, las tasas seguían aumentando, aunque a un ritmo más estatal de entre el $4 \%$ y el $6 \%$ según el mercado. Ecuador es promocionado como el destino perfecto para compradores nacionales o extranjeros que buscan jubilarse, gracias a sus bajos precios de propiedad y al costo de vida, e incluso la impresionante apreciación de los hogares y el desarrollo económico general de la última década no han cambiado esto.

Durante la última década, los precios inmobiliarios han sido empujados hacia arriba por una serie de factores. El crecimiento económico ha sido fuerte, especialmente antes de la caída en los precios del petróleo. Ecuador estaba especialmente bien posicionado con sus programas sociales, muchos de los cuales fueron estructurados para albergar a la mayor cantidad de población de bajos ingresos del país en residencias formales como sea posible.

Las urbanizaciones también son claves para la industria de los bienes raíces en Ecuador. Desde 1990, la población urbana del país ha aumentado en una tasa promedio de $2.7 \%$. (Inec, 2018). Para 2010 , el $67 \%$ de la población vivía en las ciudades. La construcción también se mantiene fuerte en el subsector inmobiliario comercial. Los hoteles en particular buscan capitalizar la creciente reputación del país como destino turístico, así como también los viajes de negocios a las principales ciudades.

\section{Desarrollo}

Además del obvio auge de la construcción en Quito y Guayaquil, las dos ciudades del país con más de un millón de habitantes, Cuenca y Manta también han experimentado auges de la construcción en la última década. Durante la última década, el acceso 


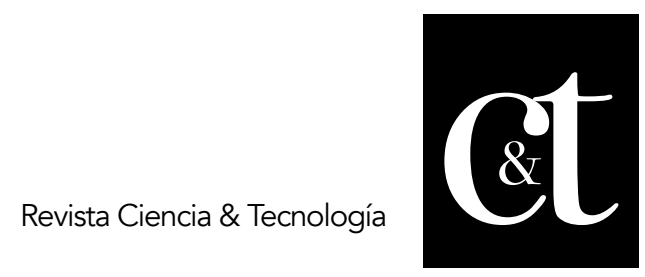

No. 22, 30 de abril de 2019

ISSN impreso: 1390 - 6321

ISSN online: 2661 - 6734

mejorado a las hipotecas ha impulsado las compras de viviendas en los centros urbanos por parte de ciudadanos privados. Mientras tanto, las grandes empresas han ayudado a sus empleados a comprar sus casas cerca del lugar de trabajo.

Otra tendencia ha sido el número cada vez mayor de multinacionales que se establecen en el país, y que a su vez han empoderado tanto a residentes locales como a extranjeros con compras de casas y alquileres de departamentos.

Sin embargo, a partir de 2014, una confluencia de eventos creó lo que ahora son importantes obstáculos para la economía de Ecuador, lo que a su vez ha afectado los precios de los activos, específicamente los bienes raíces. En julio de 2014, los precios del petróleo comenzaron a desplomarse (INEC, 2014). Al mismo tiempo, los bancos en Ecuador endurecieron el crédito.

Ecuador experimentó un aumento masivo en su oferta de dinero entre 2007 y 2014. Al mismo tiempo, los bancos de Ecuador (tanto privados como públicos) estaban expandiendo el crédito. Préstamos, con intereses a menudo en la parte superior de la adolescencia o veinte años y fuertemente garantizados se convirtieron en algo común.

A fines de 2014, los bancos comenzaron a restringir el crédito. Las líneas de crédito fueron retiradas para los constructores, las hipotecas se hicieron difíciles de obtener y los estándares de préstamos personales y comerciales mucho más estrictos. En parte debido a la contracción del crédito, el auge de la construcción que Ecuador experimentó durante la mayor parte de una década se desaceleró.

Al mismo tiempo, el gobierno anunció recortes presupuestarios: el presupuesto propuesto para 2017 fue más de \$ 5 mil millones menos que el de 2016. La reducción del gasto público colocó un lastre adicional en la economía (ASOBAN, 2016). Muchos también argumentarían las medidas fiscales y regulatorias cada vez más restrictivas. Estos eventos se reflejaron en el PIB así como en el crecimiento de la oferta monetaria, ambos prácticamente planos en 2016.

Durante la última década, Ecuador ha sufrido una serie de cambios tumultuosos. Sin embargo, hoy el país se ha convertido en un refugio acogedor para aquellos que desean retirarse o invertir aquí. El mercado inmobiliario está en aumento. En el cantón General Villamil Playas se puede disfrutar de un estilo de vida cómodo con solo una fracción de lo que gastaría en muchas partes del mundo.

Asimismo, la República del Ecuador busca proteger a los grupos vulnerables como los niños, las personas con discapacidad y las personas mayores. Estos tienen leyes especiales para proteger sus derechos que especifica el rol del sector público y privado para garantizar la Ley de Personas Mayores y se siguen sus regulaciones, el Instituto Ecuatoriano de Seguridad Social es el ente encargado de proporcionar las pensiones correspondientes a los adultos mayores jubilados.

Existen innumerables propuestas habitacionales enfocadas principalmente a personas de un rango de edad comprendida entre los 25 a 45 años, siendo este nicho de mercado realmente atractivo para el sector de la banca como para las inmobiliarias, sin embargo, existe un crecimiento ya sostenido año tras año de adultos mayores que se jubilan en sus lugares de trabajo o a su vez están en un 


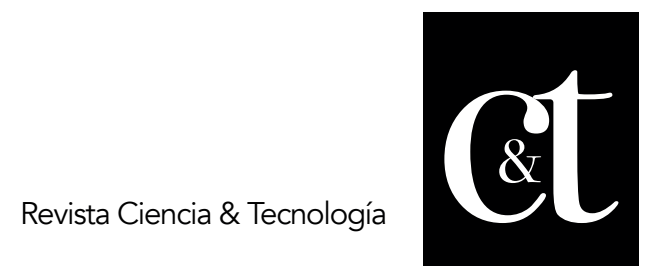

No. 22, 30 de abril de 2019

ISSN impreso: 1390 - 6321

ISSN online: 2661 - 6734

proceso de jubilación, esto también podría significar una gran oportunidad de negocio para dar una solución de vivienda a este sector que busca ya retirarse y disfrutar de la vida.

Según cifras del Instituto Ecuatoriano de Seguridad Social para el cierre del año 2016 existen aproximadamente cuatrocientos dos mil trescientos sesenta y ocho personas en condición de jubilados y otras veintiséis mil personas en trámite de jubilación, siendo las ciudades de Quito y Guayaquil las que mayor número de pensionistas mantiene, sin embargo, la ciudad de Cuenca ha logrado captar y dinamizar tanto a extranjeros como nacionales para vivir y adquirir inmuebles en sus etapas de retiro.

El poder adquisitivo que poseen los pensionistas y jubilados no ha sido totalmente aprovechados ni por las instituciones financieras ni por las empresas del sector inmobiliario para motivar proyectos o aumentar la venta de villas en las urbanizaciones que actualmente existen sobre todo en los cantones de la provincia del Guayas, teniendo en cuenta que la situación actual del país mantiene un escenario cambiante en lo social y económico, es necesario tomar en consideración desarrollar estrategias de comercialización de villas en las urbanizaciones de la provincia del Guayas.

En General Villamil Playas existen algunas urbanizaciones que se encargan de ofrecer viviendas o también llamadas villas, a cualquier tipo de persona nacional o extranjera que solo o en conjunto con su familia desean radicarse en zonas cercanas al mar, sin embargo, las mismas no han determinado sus nichos de mercados pues usan muy pocas estrategias de comercialización dirigiéndose a todas las poner sin tomar en cuenta los factores demográficos, geográficos o conductuales de los mismos.

El presente artículo científico busca desarrollar estrategias de comercialización de las villas ubicadas en las urbanizaciones del cantón General Villamil Playas. Tomando como nicho de mercado a los adultos mayores que ya reciben jubilación, para dar a conocer este producto es necesario primero realizar un respectivo estudio de campo en el que se pueda diagnosticar las preferencias de los adultos mayores, en cuanto a la idea de vivir en las zonas cercanas al mar.

Los beneficiarios del proyecto serán en primer lugar los jubilados, los mismos que tendrán acceso a obtener una casa con características adecuadas para el estilo de vida que ellos llevan, otro sector beneficiado sería la inmobiliaria, debido a su diversificación en su portafolio de productos, generando mayor rentabilidad a través de las ventas; el Estado a través de las instituciones financieras que les significaría una imagen positiva al consolidar una estrategia al otorgar casas de interés social, y los proveedores, quienes generan plazas de trabajo por la compra de materiales de construcción y la contratación de mano de obra.

Es necesario cubrir las necesidades de las personas que están jubiladas o que se encuentran en etapa de jubilación, el adquirir una vivienda con características acordes a sus necesidades, puesto esto les brindara seguridad y tranquilidad. El principal objetivo es captar este nicho de mercado considerando que estas personas son sujetas de crédito hasta los 70 años, obviamente, siempre y cuando cumpla con los requisitos exigidos por las instituciones financieras públicas y privadas que financian los proyectos habitacionales. 


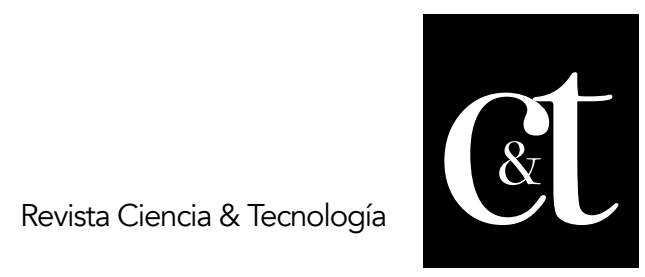

No. 22, 30 de abril de 2019

ISSN impreso: 1390 - 6321

ISSN online: 2661 - 6734

Un jubilado busca comodidad, facilidad, espacios donde poder desarrollarse como individuo y compartir con personas de iguales características y sistema de vida. Es por ello que se propone satisfacer esta necesidad con la construcción de un proyecto habitacional que permite ofrecerles casas o departamentos funcionales, casas de preferencia de una sola planta, con rampas, barandales, puertas anchas, botones de alarma ubicados estratégicamente y conectados al punto médico, áreas recreativas, áreas verdes y demás que les permite sentirse en constante actividad.

\section{Materiales y métodos}

Se realizó una investigación exploratoria dirigida a los jubilados reconocidos por el Instituto Ecuatoriano de Seguridad Social (IESS), para identificar sus necesidades, preferencias y aceptación de vivir en una urbanización, para el cierre del año 2016 existen aproximadamente 102.528 personas en condicione de jubilados en la ciudad de Guayaquil.

Una vez identificado el total de la población, se procedió a realizar el respectivo cálculo del universo muestral. Para el mismo se aplicó el método aleatorio simple, en el cual todos los elementos pertenecientes a la población tienen la misma probabilidad de ser seleccionados para la muestra. De acuerdo a la formula usada se determinó que el universo muestral fue de 384 personas.

La técnica para la recolección de datos que se usó fue la encuesta, puesto que a través de la misma se obtiene una gran fuente de información primordial para el cumplimiento del objetivo del presente artículo. A su vez, la encuesta permitirá agrupar las respuestas obtenidas de la muestra, para proceder a realizar un análisis cuantitativo de los mismos.

\section{Resultados}

Los datos de la investigación exploratoria indican que los jubilados de la ciudad de Guayaquil indicaron en una gran mayoría que si les gustaría vivir en una urbanización cerca del mar, puesto que buscan paz en sus retiros, siendo el mar el lugar más común en el que desean vivir.

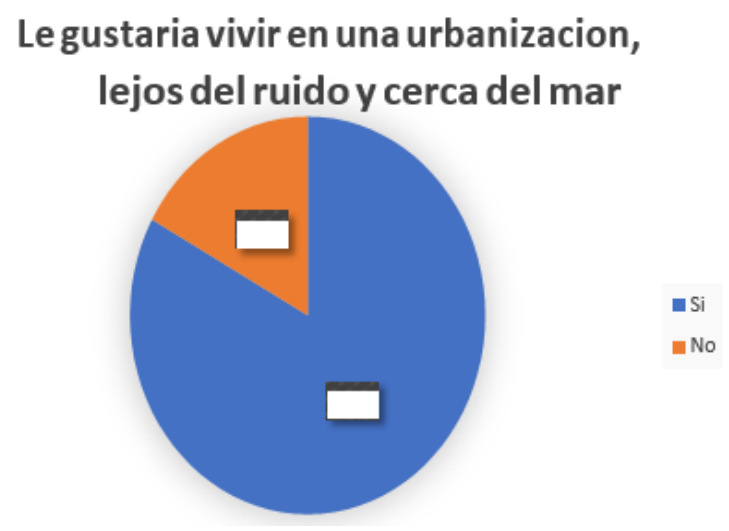

\section{Gráfico 1. Gusto por vivir en una urbanización}

Fuente: Elaboración propia

Así mismo indicaron que el cantón costero de su preferencia seria General Villamil 
Playas, puesto que, en aquel lugar, la afluencia de público no es igual que en otros cantones como Salinas o Puerto López, siendo así consideran a Playas como el cantón más recomendable para empezar su vida de retiro. Cerca de Playas se ubicó el cantón Salinas, los que decidieron por este cantón manifestaron que lo eligen por su diversidad de playas y por gastronomía.

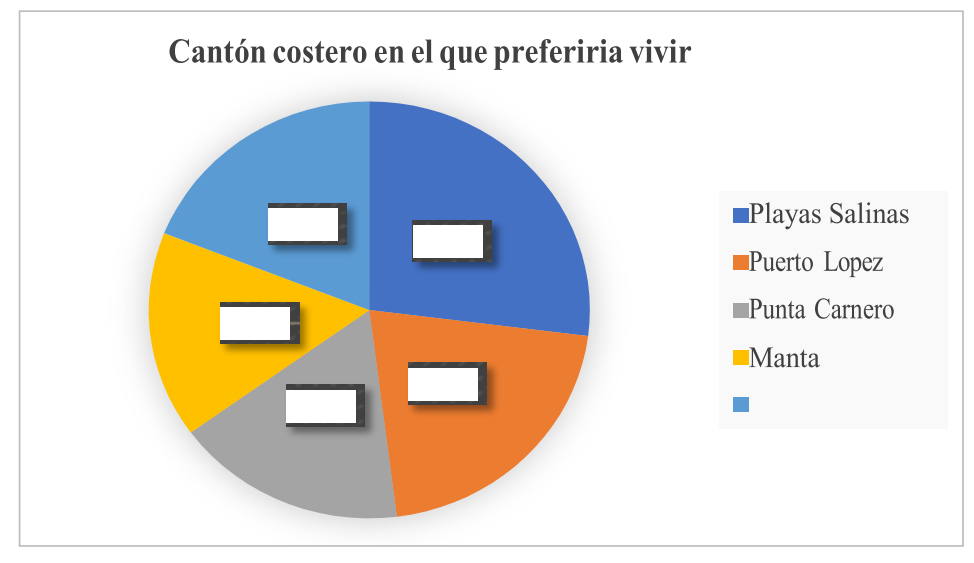

\section{Gráfico 2. Cantón costero de preferencia}

Fuente: Elaboración propia

Se pudo conocer que en un gran numero la mayoría de personas jubiladas, reciben una pensión entre un rango de $\$ 800$ a $\$ 1200$ lo que pone en evidencia el poder adquisitivo que tienen los mismos, el cual no ha sido debidamente explotado por los diferentes sectores del país como el bancario o el inmobiliario. Otro gran porcentaje, pero menor al mencionado, fue el menor a $\$ 800$ teniendo como promedio un ingreso casi semejante a los $\$ 700$ mensuales, siendo esta la escala con menor rango en esta pregunta.

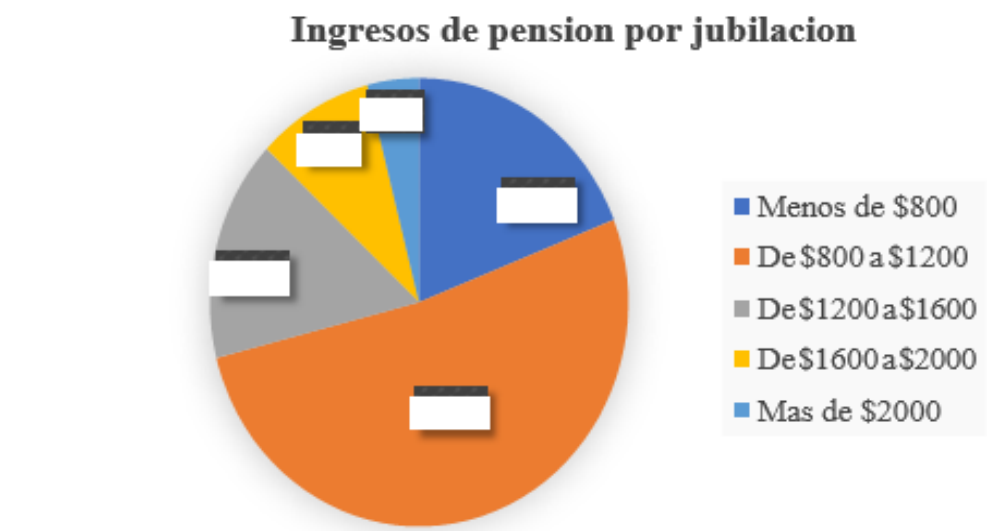

\section{Gráfico 3. Ingresos por pensión de jubilación}

Fuente: Elaboración propia

La gente jubilada, se podría decir, que son de gustos sencillos. Pues la gran mayoría de encuestas resolvió por responder que prefieren una vivienda de un solo piso, muchos porque viven solos o con sus cónyuges por lo que no necesariamente necesitarían una villa de grandes proporciones. La segunda opción más seleccionada, pero con mucha menor diferencia a la anterior fue una vivienda de dos pisos, en 
muchos de estas respuestas como razón a la misma se decía que viven con más de 3 personas.

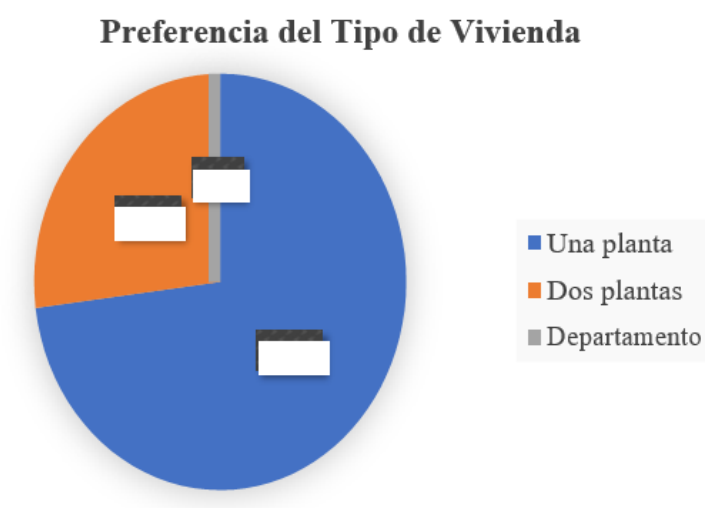

\section{Gráfico 4. Preferencia de tipo de vivienda}

Fuente: Elaboración propia

Para los encuestados si ellos estuvieran viviendo en una urbanización, es indispensable, que la misma cuente principalmente con restaurantes, pues muchos viven solos y necesitan un lugar con comida sana al que puedan asistir para mantener una dieta nutritiva. En segunda instancia, es necesario para que ellos que la urbanización cuente con algunas farmacias, pues en personas con una edad superior a los 65 años se necesitan de más cuidados y tener farmacias cercas es primordial para mantener tranquilo a las personas. También mencionan que un centro médico cercano sería un plus muy grande para la selección de una urbanización y más si la misma estaría ubicada en el cantón General Villamil Playas, pues si en algún momento llegasen a tener una descompensación en sus salud, se tendría un doctor experto en el trato a estas personas, para que se les pueda brindar la atención necesaria con un servicio de la más alta calidad.

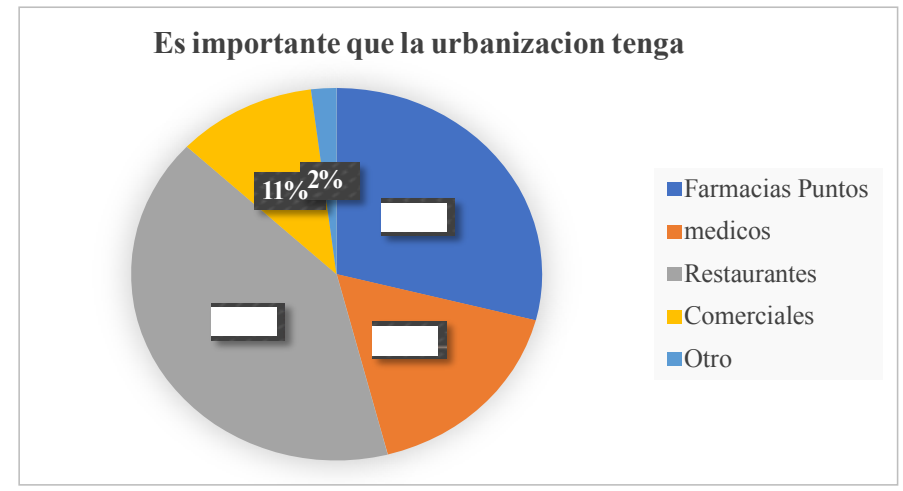

\section{Gráfico 5. Lugares importantes que debe tener la urbanización \\ Fuente: Elaboración propia.}

La gran mayoría de los encuestados, indicaron estar dispuestos a invertir en una villa dentro de una urbanización que cumpla con todas las necesidades, requerimientos y deseos de los jubilados a quienes se les ha realizado esta investigación exploratoria, dejaron claro que necesitan recibir más información sobre las urbanizaciones del 


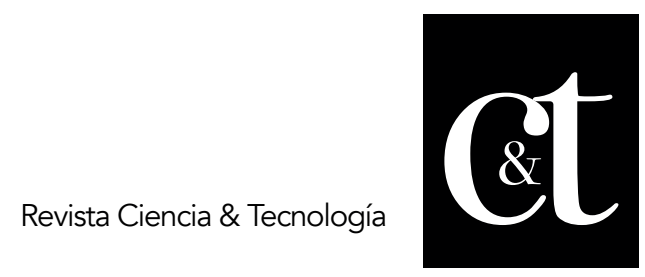

No. 22, 30 de abril de 2019

ISSN impreso: 1390 - 6321

ISSN online: 2661 - 6734

cantón Playas por lo que es necesario aplicar estrategias de comercialización para este tipo de bien que ofrecen las urbanizaciones.

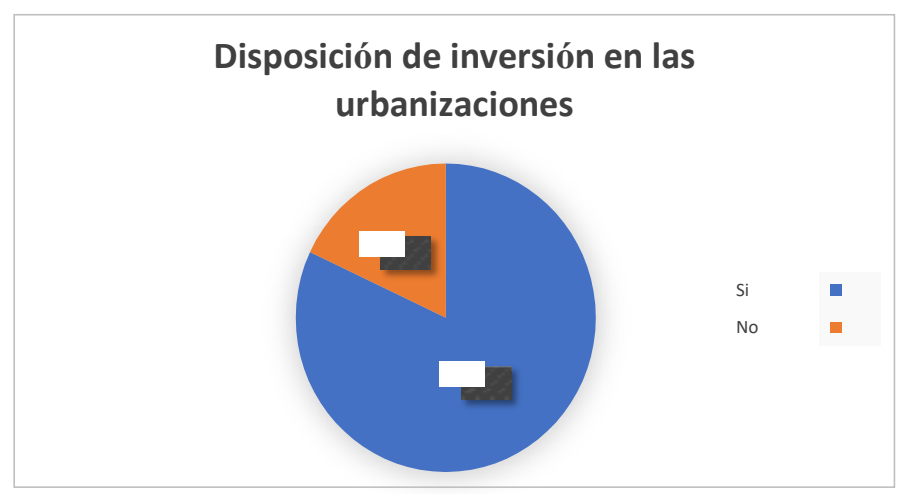

\section{Gráfico 6. Disposición de invertir en urbanizaciones}

Fuente: Elaboración propia.

\section{Discusión}

En marketing, los planes efectivos comienzan con objetivos de utilidad (Tennyson y Ray, 2005). Todas las actividades de comunicación deben apoyar las metas y objetivos generales de la organización. Primero, la marca necesita definir los objetivos de la organización. Una vez que los objetivos de la organización están claramente definidos, se pueden desarrollar los objetivos y las metas de comunicación.

En primer lugar, es muy importante que los objetivos que las empresas eligen sean cuantificables. Deben tener un plazo corto o largo, ser claros y precisos, ser mensurables y finalmente ser realistas (Drucker, 1954). Finalmente, también es importante mencionar que los objetivos de comunicación tienen funciones específicas 1) Coordinar y promover el plan de comunicación; 2) Proporcionar un criterio para tomar decisiones hacia campañas múltiples; 3) Hace posible evaluar los resultados de la comunicación.

En los medios ferozmente competitivos de hoy en día, no hay escasez de formas potenciales de comercializar bienes raíces. Sin embargo, como cualquier otra disciplina de marketing, ponerse delante de los clientes correctos se hace cada vez más difícil. A medida que los agentes inmobiliarios y los propietarios se dan cuenta de que la empinada batalla cuesta arriba, necesitan recurrir a profesionales para que los ayuden en este campo de marketing en línea altamente complejo e intrincado.

Sin embargo, por muy lucrativo que sea el mercado de bienes raíces para los potenciales reparadores y agencias que buscan trasladar las casas rápidamente, existen algunos métodos comprobados para el éxito en los negocios, que les permiten vender una casa de manera más rápida y eficiente que antes mediante el uso de las estrategias correctas de mercadeo de bienes raíces.

Las fotos siempre han sido importantes cuando se trata de bienes raíces. Sin embargo, no hay tantos propietarios ni agentes inmobiliarios que comprendan la importancia de este concepto único cuando se trata de promocionar una casa o apartamento en línea. La verdad es que la gente juzgará un libro por su portada, 


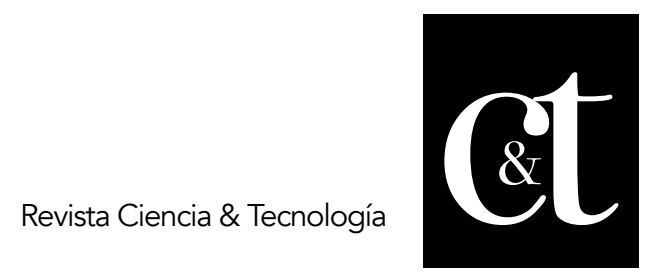

No. 22, 30 de abril de 2019

ISSN impreso: 1390 - 6321

ISSN online: 2661 - 6734

especialmente cuando navega a través de cientos o miles de listas.

Lo que quiere es algo que será atractivo y llamará la atención de una persona que está revisando innumerables listados en su área. Tiene que transmitir adecuadamente un espacio de calidad a través de estándares profesionales con el uso de cosas como un lente gran angular y una edición cuidadosa (pero no demasiado modificada) de Photoshop.

Un análisis e identificación del cliente determina qué hace que el consumidor objetivo sea único. Esto incluye características demográficas como la edad, el nivel de educación, el sexo, los ingresos y la ubicación geográfica. Una vez que la marca sabe a quién se dirigirá, también debe determinar cómo va a llegar a su público. Un análisis identifica los medios que una audiencia probablemente usará, incluyendo revistas específicas, periódicos, programas de televisión o internet.

La planificación es hoy en día prevalente. Esta es una tendencia creciente en el campo de la comunicación. En lugar de comenzar con el objetivo general, mensaje o estrategia, el plan comienza con la audiencia. Cada audiencia, una vez identificada, tiene un plan de comunicación completo construido a su alrededor. De acuerdo con el enfoque centrado en la audiencia, cada audiencia merece su propio conjunto de objetivos, estrategias, mensajes y tácticas. Un error fatal común en los planes de comunicación es la imposibilidad de identificar audiencias. La razón principal para identificar audiencias es que los diferentes grupos requieren diferentes tipos de información, en diferentes momentos y con diferentes áreas de enfoque.

Los planes de comunicación de marketing pueden diversificar el contenido de sus mensajes sobre la marca y la ejecución creativa. Una comunicación puede contener una gran cantidad de información relacionada con la marca o ninguna, la información relacionada con la marca puede enfocarse en aspectos tangibles o aspectos intangibles del producto, así como también en el nombre de la marca en sí.

En términos de ejecución creativa, la información relacionada con la marca puede transmitirse en virtualmente un número infinito de diferentes. Es importante mencionar que los mensajes, utilizados en la comunicación, permiten a todas las organizaciones enfocar su comunicación de tal manera que les permita a sus audiencias comprender y retener lo que la organización intenta transmitirles (Tennyson y Ray, 2005). Por lo tanto, las comunicaciones de marketing pueden variar enormemente en la cantidad y naturaleza de la información relacionada con la marca, así como en la información de ejecución (Lane, 2001).

La importancia de mantener una presencia en línea es vital para la longevidad de los profesionales inmobiliarios tradicionales. Chatterjee et al. (2013) sugirieron implementar sistemas de información, diseñados con un propósito particular de organización, social o económico, demostrando ser más beneficiosos para todos los usuarios y personas involucradas en el entorno. Los avances en la tecnología y el mayor uso de Internet para llevar a cabo compras e investigación de productos continúan afectando a todas las organizaciones. Las redes sociales, las comunidades virtuales y el comercio electrónico continúan evolucionando para satisfacer las necesidades de cambio de los consumidores 


\section{Conclusiones}

Los profesionales inmobiliarios continúan ganando presencia en línea, pero la competencia de sitios web independientes y fáciles de usar que permiten a los propietarios crear materiales de marketing y enumerar la propiedad como a la venta por el propietario ha ralentizado el progreso. Estudiosos como Richardson y Zumpano (2014) y Yuan, Lee, Kim y Kim (2013) estudiaron el comportamiento de búsqueda y abordaron el efecto de la actividad de Internet en las ventas de viviendas. Los patrones de comportamiento de búsqueda que encontraron una mayor eficiencia en los sitios web inmobiliarios en línea y los patrones identificados podrían utilizarse para desarrollar una guía para el uso profesional inmobiliario en estrategias de comercio electrónico (Yuan et al., 2013).

Es posible que los gerentes de las empresas inmobiliarias residenciales tradicionales no tengan la capacidad de competir con los sitios web de bienes raíces de comercio electrónico. Los profesionales de bienes raíces sucumben a la presión de competir y mantenerse innovadores con nuevas estrategias (Love, Goh, Hogg, Robson, \& Irani, 2011). Por lo tanto, era necesario explorar las estrategias que los gerentes de la organización de bienes raíces residenciales tradicionales podrían implementar para competir con las organizaciones de bienes raíces de comercio electrónico.

Una vez que se revisó el estudio de mercado, se tienen grandes ventajas como el hecho de que el gobierno esté dando facilidades a través de las instituciones financieras como la CFN para los constructores y el BIESS para los clientes, dando tasas de interés bajas que permiten el acceso a los créditos correspondientes; que si bien es cierto la tendencia normalmente es a comprar villas con construcción tradicional pero los clientes de este segmento entienden que la tecnología usada en este tipo de construcción es incluso superior a la tradicional y sobre todo que siendo este tipo de urbanización inédito en el mercado va a tener una gran acogida según las encuestas que se realizaron.

Es necesario cubrir las necesidades de las personas que están jubiladas o que se encuentran en etapa de jubilación y que puedan adquirir una vivienda con características acordes a sus necesidades, puesto esto les brindara seguridad y tranquilidad. El principal objetivo es captar este nicho de mercado considerando que estas personas son sujetas de crédito hasta los 70 años, obviamente, siempre y cuando cumpla con los requisitos exigidos por las instituciones financieras públicas y privadas que financian los proyectos habitacionales.

\section{Recomendaciones}

Se les recomienda a las urbanizaciones presentes en el cantón General Villamil Playas que haya una innovación en el producto y en el segmento del grupo meta y que se concentre en un nuevo nicho de mercado en la ciudad de Guayaquil. Los pensionistas brindarán la oportunidad de que las urbanizaciones del cantón General de Villamil Playas puedan aumentar sus ingresos y masificar sus servicios, posicionar esta nueva marca y así, ganar otro nicho de mercado que competirá de acuerdo con las solicitudes de lo mismo.

Si las urbanizaciones e inmobiliarias del cantón general Villamil Playas quieren permanecer en el mercado e incluso construir más etapas de estas características, primero debe cumplir con los requisitos y deseos acordados y conocidos en la encuesta de campo a los clientes potenciales en el documento legal firmado, para 


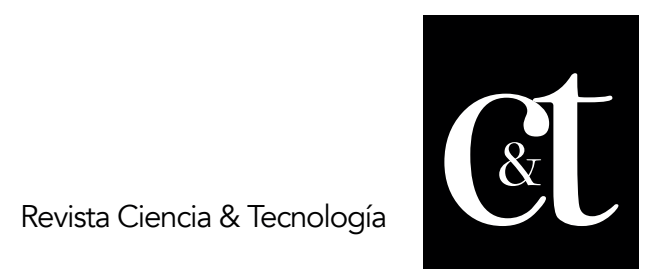

No. 22, 30 de abril de 2019

ISSN impreso: 1390 - 6321

ISSN online: 2661 - 6734

ganar la confianza del cliente final.

\section{Referencias bibliográficas}

ASOBAN. (2016). Boletín Macroeconómico. Guayaquil, Ecuador: Asoban.

Banco Central del Ecuador. (12 de Enero de 2015). Recuperado el 03 de Septiembre de 2015, de http://www.bce.fin.ec/index.php/component/k2/item/310producto-interno-bruto

CCG. (2018). Revista Digital de CCG. Guayaquil, Ecuador: CCG.

Chatterjee, S., Merhout, J. W., Sarker, S., y Lee, A. S. (2013). Un examen de la hipótesis del mercado electrónico en la industria hipotecaria de los Estados Unidos: un estudio de caso deductivo. Tecnología de la información y personas, 26, 4-27.

Drucker, P. (1954). The practice of management. New York, USA: Harper \& Row.

Grande, I., \& Abascal, E. (2011). Fundamentos y técnicas de investigación comercial. Madrid: Gráficas Dehon.

INEC. (2014). INEC Esadísticas. Quito, Ecuador: INEC.

INEC (2018). INEC Estadísticas. Quito, Ecuador: Inec.

Marketing Publishing Center. (2012). El plan de negocios. Barcelona: Díaz de santos.

Lane, K. (2001). Mastering the marketing communications: Micro and macro perspectives on integrated marketing communication programs. Journal of marketing management. V. 17(7-8). Pp. 819-847.

Love, P., Goh, Y., Robson, S. \& Irani, Z. (2011). Burnout and sense of coherence among real estate brokers. Safety Science. Vol. 49. Pp. 1297-1308.

Moreno, M. (2011). Introducción a la metodología de la investigación educativa. Madrid: Progreso.

Richardson, H. \& Zumpano, L. (2012). Further assessment of the efficiency effects of internet use in home search. Journal of real estate research. Vol. 34(4), pp. 515548.

Talaya, E. (2011). Principios de marketing. Madrid, España: ASIC.

Tennyson, P. \& Ray, K. (2005). Creating a strategic communication plan that gathers no dust. Journal AWWA. V. 97(1), pp. 48-57.

Yuan, X., Kim, S. \& Lee, J. (2013). Toward a user-oriented recommendation system for real estate websites. Information Systems. Vol. 38(2), pp. 231-243. 